\title{
Kinematic analysis of post office employees' workstations
}

\author{
Francesco Draicchio ${ }^{\mathrm{a},{ }^{*}}$, Alessio Silvetti ${ }^{\mathrm{a}}$, Federico Forzano $^{\mathrm{a}}$, Sergio Iavicoli $^{\mathrm{a}}$ and Alberto Ranavolo ${ }^{\mathrm{a}}$ \\ ${ }^{a}$ Department of Occupational Medicine, INAIL formerly ISPESL, Via F. Candida 1, 00040 \\ Monte Porzio Catone, Italy
}

\begin{abstract}
This study analyzed a post office clerk's tasks, comparing two workstation models. The clerk was facing the client in one, and seated at $45^{\circ}$ to the counter in the other. We analyzed the most frequent tasks and those presenting the most critical points: 1) payment of a postal order; 2) accepting a registered letter, breaking them down into subtasks. We used an optoelectronic system for kinematic analysis, and calculated the range of motion of the trunk and arms in the three spatial planes. The $45^{\circ}$ position required less torsion of the trunk and head when using the printer, placed to the left of the employee. A larger worktop improved the workstation, leaving more room for equipment and allowing the worker to sit frontally to the monitor. However, this solution involved a shorter distance between the worker and the client with longer extension of the shoulder and elbow and less trunk flexion. These findings suggested a modification in the layout that shortens the distance between the worker and client.
\end{abstract}

Keywords: ergonomic redesign; biomechanics; posture

\section{Introduction}

The equipment on a work station must all be placed so that the operator can reach it easily. In addition, in the services sector, such as banks and post offices, or in shops and supermarkets, ergonomic planning for a work station must take account not only of the equipment, but of the movements involved in interactions with clients. Any changes in position - either of equipment or for interactions not only affects productivity but also has implications for biomechanical overload. Sengupta [6] noted that energy expenditure was closely related to the size of the reach envelope. Early studies investigated reach arcs in static conditions, without taking account of trunk movements $[1,3,5,7]$ but more recent ones have also considered dynamic conditions [4].

The post office offers a classic work station where the clerk, with a computer videoterminal (VDT) in front, has to interact with the client. There are various strategies for this layout. We examined the post office employee's reach situation and interface with clients, comparing two work station models. One was linear, with the clerk facing the client and the monitor at a $45^{\circ}$ angle to the main axis of the work top.

This is the layout used in Italian post offices, but it presents some problems for VDT work, as it is not always easy to line up the monitor, keyboard and worker comfortably. The position of the monitor is a key feature in planning a VDT workstation, as it directly influences the employee's posture and visual work [8].

We also tested a new, angular worktop, using the same structure, to obtain at limited cost a workstation that is better adapted to VDT work and the clientemployee interface. The prototype worktop is in two sections with a $90^{\circ}$ corner, and the clerk sits at $45^{\circ}$ to the client, but in line with the monitor and keyboard.

We compared the range of movements of the employee's head, trunk, shoulders and elbows while doing different jobs on the two worktops. Focus was mostly on the client-worker contact and on access to the main peripherals (printer and Roller Cash), to study how the ergonomic intervention affected these operations. We also did a kinematic analysis comparing the same tasks done at the two workstations.

\footnotetext{
*Corresponding author. E-mail: f.draicchio@inail.it.
} 


\section{Material and methods}

\subsection{Participants}

Five experienced male employees with more than five years of working experience were enrolled.

Their average (SD) age, height and weight were respectively 37 years $(4.1), 175 \mathrm{~cm}(2.8)$ and $78 \mathrm{~kg}$ (8.1). They voluntarily performed the study tests in a laboratory. None of them had any history of either musculoskeletal disorders or neurological diseases.

All gave written consent after they had received a full explanation of the study procedure.

\subsection{Instrumentation}

An opto-electronic motion analysis system was used (Fig. 1) (SMART-E System, BTS, Milan, Italy), [2] consisting of eight infrared ray cameras, operating at $120 \mathrm{~Hz}$. Anthropometric data were collected for each subject and 15 spherical markers coated with aluminum powder reflecting material were placed over prominent bony landmarks: two were placed symmetrically on the right and left temporal crests, one on the middle frontal region, one on the skin over the spinous processes of the 7 th cervical vertebra, one on the skin over the spinous processes of the 2 nd thoracic vertebra, two over the right and left acromions, two on the right and left olecranon, two over the right and left ulnar styloid process, two over the right and left radial styloid process, and two over the last phalanxes of the right and left forefingers.

Two markers were also placed on the edge of the chair to calculate the trunk torsion and chair rotation.

As the chair was fitted with a lumbar support we could not place a marker at the sacrum, so trunk flexion data only refer to the upper body. The markers were attached in such a way that they could not fall out of place during data acquisition.

To calibrate the system before the first data capture, kinematic data were acquired and digitized with a sampling rate of $120 \mathrm{~Hz}$. Spatial accuracy was $<0.5$ $\mathrm{mm}$ in the calibrated volume, which was approximately $7 \mathrm{~m}$ long, $4.00 \mathrm{~m}$ wide and about $2.50 \mathrm{~m}$ high.

\subsection{Experimental procedures}

We analyzed a post office employee's tasks, comparing two work station models. Figure 2 shows the measurements and design of the two workstations, the grey rectangle indicating the linear desktop which

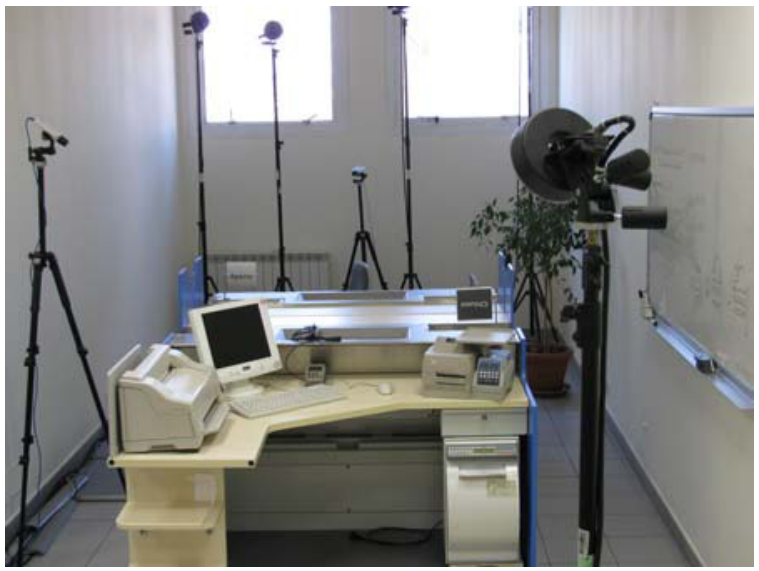

Figure 1

The angular desk set up in the laboratory. In the right foreground is one of the eight infrared ray cameras

measures 150 by $65 \mathrm{~cm}$. The other is the corner model. The clerk sits facing the client in the linear workstation, and at $45^{\circ}$ to the counter in the angular one. In both layouts the employee deals with the client in the same position, marked on both counters by a metal tray fitted into the frame at a height of 105 $\mathrm{cm}$ from the floor. The Roller Cash machines are also installed in the same position in the two arrangements, to the worker's right. The printers are in exactly the same position too, but in the linear set-up they stand on an extra unit alongside the worktop (Figure 3).

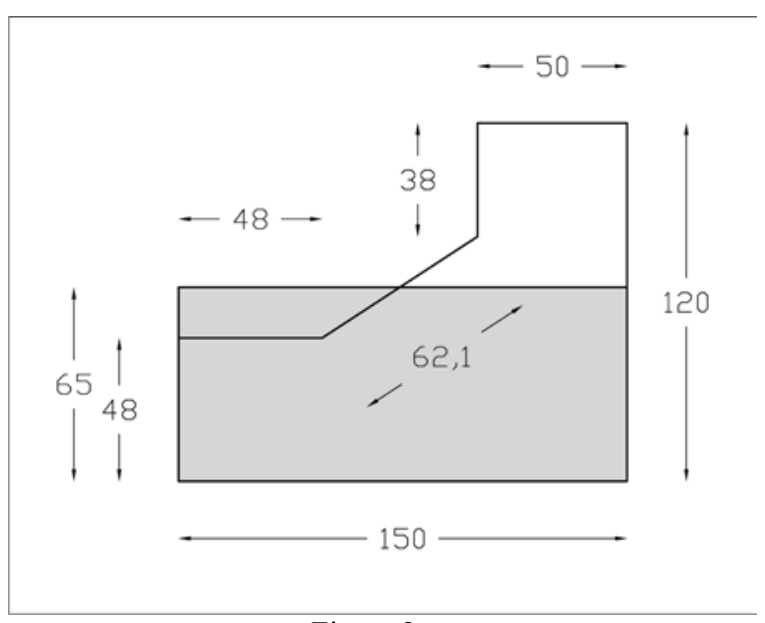

Figure 2

Design and measurements of the two worktops, the grey area indicating the linear set-up, shown overlying the plan of the angular top. 


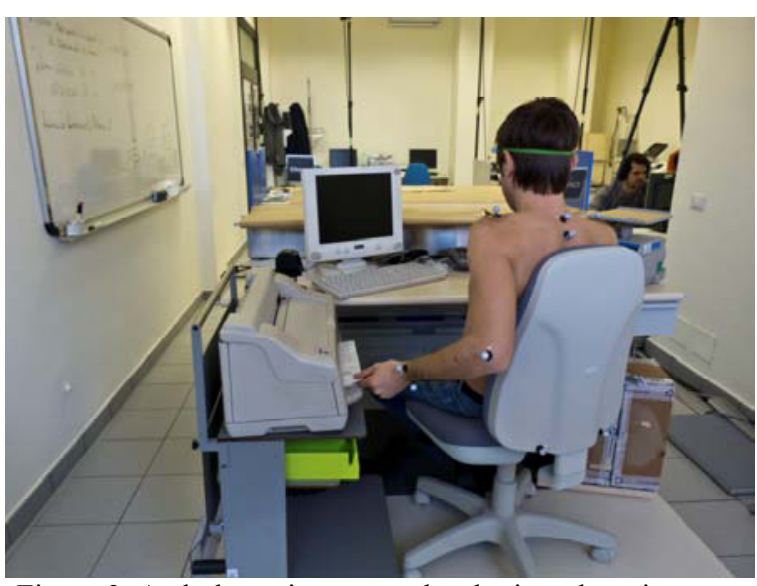

Figure 3. A clerk putting a postal order into the printer.

The markers for the kinematic study can be seen.

We selected five people, who were trained to do the study task. They were selected on the basis of their arm measurements, which had to be between the 45th and 55th percentiles. Before starting the task each person adjusted the seat to the height they preferred. Each subject repeated the tasks 3 times. We analyzed the most frequent tasks and those presenting the most critical points: 1) payment of a postal order; 2) accepting a registered letter. We broke down these two operations into subtasks: 1a) the employee takes the postal order form and client's debit card; 1b) the employee takes the cash from the tray; 1c) the postal order is placed in the printer; 1d) the debit card is accepted; 1e) the receipt is inserted in the printer; 1f) the receipt is taken out of the printer and handed to the client; 1g) banknotes are run through the Roller Cash machine; $1 \mathrm{~h}$ ) the notes are taken from the Roller Cash and the client is given change. 2a) The registered mail form and letter are taken from the tray and the envelope is weighed; 2b) the letter is taken off the balance, labeled and placed in the special envelope; 2c) the registered mail bar code is read and inserted in the printer.

We then made a kinematic study of subtasks 1a, $1 \mathrm{~b}, 1 \mathrm{c}, 1 \mathrm{e}$ and $1 \mathrm{~g}$, which are the most interesting steps from the viewpoint of the interaction between the worker and the client, and access to the peripherals (printer and Roller Cash).

\subsection{Kinematic data computing}

After a frame-by-frame tracking procedure (Smart Tracker, BTS, Milan, Italy), to assign a label to each marker, data were processed using Analyzer software (Smart Analyzer, BTS, Milan, Italy). The $\mathrm{x}$-axis of the local Cartesian coordinate system lay on the subject's sagittal plane. The employee began each movement in the same position (seated in front of the client in the linear workstation and in front of the monitor in the other). The kinematic data recorded included: torsion, flexion and lateral bending of the trunk, horizontal head rotation, abduction-adduction and flexion of left and right shoulder, flexionextension of right and left elbow. For each angle, the range of motion (ROM) was calculated as the difference between the maximum and minimum values during the different tasks. The kinematic signals were filtered using a $5 \mathrm{~Hz}$ Low-Pass Filter.

\subsection{Statistical analysis approach}

All analyses were done using SPSS Statistics 17.0 software (SPSS Inc., Chicago, IL, USA). For each ROM, the mean and standard deviations (SD) were calculated. To compare the means we used Student's t-test. $P$-values less than 0.05 were considered statistically significant.

\section{Results}

Table 1 lists the mean ROMs $( \pm \mathrm{SD})$, with $P$-values less than 0.05 in bold type. The first section shows the data for the task in which the clerk takes the postal order form and client's debit card. In the new, angular workstation trunk flexion, referring only to the upper body, was significantly less, only about a quarter of that in the linear layout. At the same time shoulder flexion was slightly but significantly larger, making on the whole for a better reach arc. Elbow flexion was slightly but not significantly greater in the linear workstation.

Then when the employee reaches over to take the cash from the tray, trunk flexion at the linear workstation was again double that at the angular desk. Consequently the shoulder flexion was wider at the angular desk. Elbow flexion at the angular desk was also considerably greater than at the linear workstation. All these differences were significant.

In step 1c (Table 1) when the clerk has to put the postal order in the printer, trunk torsion was more than double in the linear layout compared to the angular setting, the difference being highly significant.

On the transverse plane head rotation on the trunk at the linear worktop was nearly double that at the angular desk though the difference did not reach significance. 
If we add all up the transverse plane data for the trunk and head rotation, the difference between the sums for the mean ROM is 27 degrees, to which we must add the greater rotation of the seat, which amounted to about $10^{\circ}$ at the linear desk but was negligible with the angular layout.

In the step where the receipt is inserted in the printer there were again significant differences between the trunk and head rotation in the two workstations (Table 1e). The sum of the rotations was once again larger for the linear layout: $17.6^{\circ}$ vs. $11.3^{\circ}$, and $6.9^{\circ}$ vs. $2.6^{\circ}$, with the rotation of the chair adding about $10^{\circ}$, whereas it was negligible for the angular worktop.

The data for the step where banknotes are run through the Roller Cash machine (Table 1g) indicate the ROM for trunk torsion and rotation of the head on the trunk, always towards the worker's right-hand side, i.e. in the opposite direction to the torsions required for the preceding step. In fact, the torsions at the angular workstation were slightly larger for the trunk and for rotation of the head on the trunk ,but only the trunk rotation difference was significant.

The rotation of the chair, again around $10^{\circ}$, must always be added to the head and trunk rotation at the angular desk, whereas it was negligible for access to the Roller Cash machine in the linear setting. Table 1

Range of movements (degrees) in the sagittal plane (1a and 1b) and transverse plane: left side (1c and 1e) and right side (1g) during different subtasks

\begin{tabular}{|c|c|c|c|}
\hline & Angular & Linear & \\
\hline & Mean \pm SD & Mean \pm SD & $P$-Value \\
\hline \multirow{4}{*}{$\begin{array}{l}\text { 1a) the employee } \\
\text { debit card } \\
\text { trunk flexion } \\
\text { right shoulder flex } \\
\text { right elbow flex-ext }\end{array}$} & akes the pos & order form & ind client's \\
\hline & $2.9 \pm 1.2$ & $11 \pm 1.3$ & 0.000007 \\
\hline & $50.9 \pm 4$ & $46.4 \pm 1.7$ & 0.0493 \\
\hline & $59.7 \pm 8.1$ & $60.8 \pm 8.8$ & 0.8422 \\
\hline \multicolumn{4}{|c|}{ 1b) the employee takes the cash from the tray } \\
\hline trunk flexion & $5.6 \pm 1.3$ & $11.5 \pm 1.3$ & 0.00009 \\
\hline right shoulder flex & $52.1 \pm 1.3$ & $45.8 \pm 0.8$ & 0.000005 \\
\hline right elbow flex-ext & $78.1 \pm 2.1$ & $61.7 \pm 7.6$ & 0.0016 \\
\hline \multicolumn{4}{|c|}{ 1c) the postal order is placed in the printer } \\
\hline trunk torsion & $10.9 \pm 2.6$ & $28.2 \pm 1.6$ & 0.000001 \\
\hline head torsion & $13.8 \pm 5.9$ & $23.4 \pm 8.2$ & 0.0663 \\
\hline \multicolumn{4}{|c|}{ 1e) the receipt is inserted in the printer } \\
\hline trunk torsion & $11.3 \pm 1.4$ & $17.6 \pm 1.1$ & 0.00005 \\
\hline head torsion & $2.6 \pm 0.1$ & $6.9 \pm 2.1$ & 0.0018 \\
\hline \multicolumn{4}{|c|}{ 1g) banknotes are run through the Roller Cash machine } \\
\hline trunk torsion & $21 \pm 0.7$ & $17.8 \pm 1.4$ & 0.0018 \\
\hline head torsion & $52.8 \pm 4.9$ & $51.9 \pm 13.4$ & 0.8913 \\
\hline
\end{tabular}

Figures 4 and 5 give two examples of threedimensional reconstruction of the model, the tips of the large triangle shaded in grey indicating the two acromial spines - where the arms start - and the spin- ous process of the seventh cervical vertebra. The smaller triangle indicates the head and the straight line between two free markers refers to the position of the chair.

Figure 4 illustrates the step where the clerk at the angular desk puts the postal order into the printer.

There is no significant rotation of the trunk, but the head turns significantly. Figure 5 refers to the same step, but with the clerk at the linear desk; here there is substantial torsion of the trunk in relation to the chair, and significant rotation of the head.

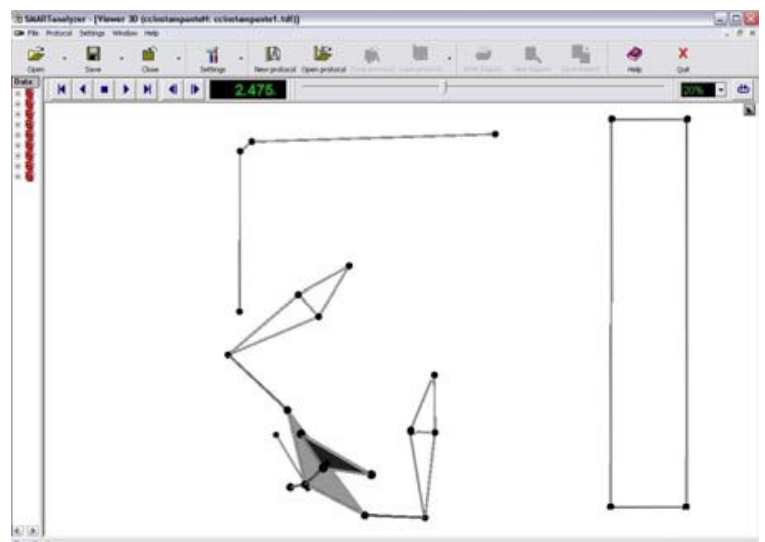

Figure 4. Kinematic model of a clerk at the angular workstation, putting a postal order into the printer. The large triangle shaded in gray indicates the trunk and the smaller black one the position of the head. Trunk torsion was negligible but there was appreciable rotation of the head. The rectangular area represents the clientworker interface.

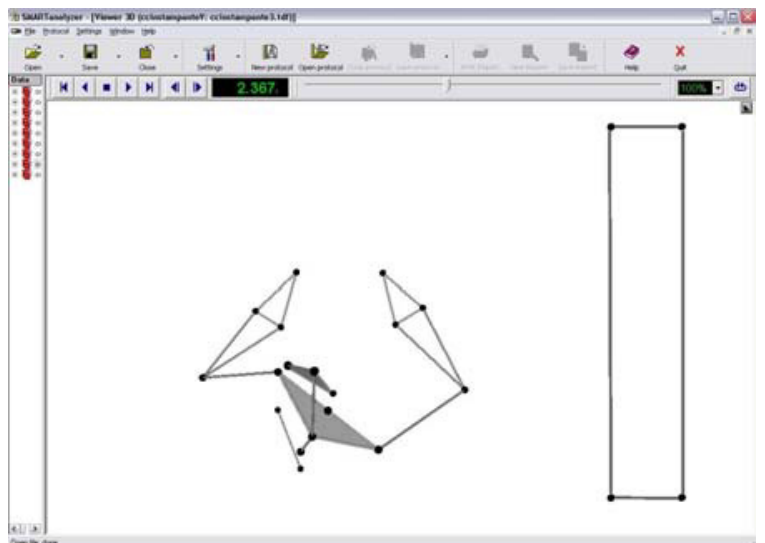

Figure 5. Kinematic model of a clerk at the linear workstation, putting a postal order into the printer. The large triangle shaded in gray indicates the trunk and the smaller black one the position of the head. Trunk torsion was substantial, as shown by the angle to the straight line passing through the markers on the chair. There was appreciable inclination of the head. 


\section{Discussion}

Compared to the linear workstation, the $45^{\circ}$ position in the angular layout required less torsion of the trunk and head when using the printer, placed to the left of the clerk, and the Roller Cash machine. In addition, despite the larger worktop, less trunk flexion was needed for interaction with clients. This was due to the different contributions of the shoulder and elbow at this distance and height $(105 \mathrm{~cm})$ of the plane of contact. The larger angular work counter meant there was more room for equipment, and the clerk could work frontally to the monitor and keyboard.

Kinematic findings gave useful information on the ROM and reach envelope for post office employees.

The opto-electronic motion analysis system gave objective data on the critical points of the workstation, which is still at the prototype stage; this is not possible with the usual standardized protocols, which are based on subjective observations so errors in quantification are inevitable. With the opto-electronic system, unlike simulation software that uses virtual settings, we were able to check the true interaction between the worker and the layout. From the data given by this system we could extract useful information not only to help occupational medicine specialists manage healthcare protocols, but also for other people involved in prevention, to draw up a fuller, real assessment of the risk of biomechanical overload.

Designers and planners could also use the data to study work layouts focused on the worker and the process.

In the present case the angular counter proposed as a means of overcoming the critical points of today's linear desktops respects ergonomic standards for VDT work, while at the same time improving the worker's performance, and consequently also productivity. It reduces trunk flexion in interactions with clients, and trunk torsion when using the printer or Roller Cash. With the shorter distance between the employee and the client, the shoulder extension and the elbow flexion work together to reduce the flexion of the trunk.

To make this type of adaptation modular, we propose a modification to the angular prototype, illustrated in Figure 6. This solution, which we shall shortly be testing, should optimize the relationship between trunk flexion and shoulder and elbow exten- sion, depending on each worker's body proportions, in contacts with clients.

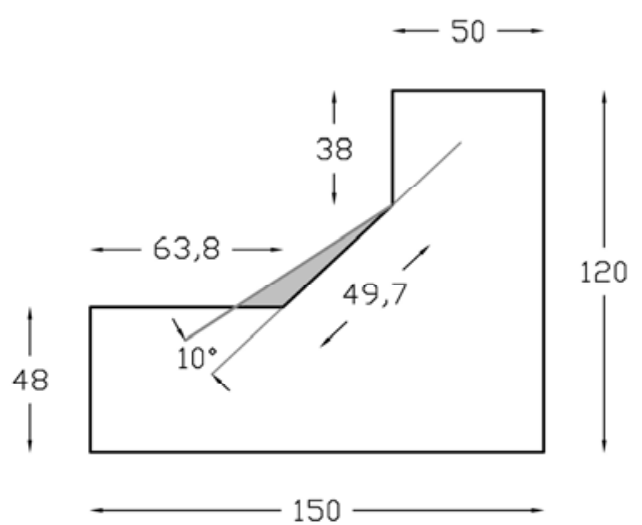

Figure 6. The modification suggested for the angular prototype: the grey area indicates the part of the worktop we intend to eliminate, to shorten the distance between clerk and client, and optimize the relationship between trunk flexion and shoulder and elbow extension, depending on each employee's body proportions.

\section{References}

[1] B. Das and D.N. Behara, Determination of the normal horizontal working area: A new model and method. Ergonomics, 38 (1995), 734-748.

[2] G. Ferrigno and A. Pedotti, ELITE: a digital dedicated hardware system for movement analysis via real-time TV signal processing. IEEE T. Bio-Med. Eng. 32 (1985), 943-950.

[3] C.M. Haslegrave, Characterizing the anthropometric extremes of the population, Ergonomics, 29 (1986), 281-301.

[4] G.T. Holman, J. Davis, and S. Maghsoodloo, The effects of dynamic movement on seated reach arcs. Ergonomics, 51 (2008), 691-701.

[5] J.T. Roth, M.M. Ayoub and C.G. Halcomb, Seating console and workplace design: seated operator reach profiles, in Proceedings of the Human Factors Society, 21st Annual Meeting, (1977) 83-87.

[6] A.K. Sengupta and B. Das, Determination of worker physiological cost in workspace reach envelopes. Ergonomics, 47 (2004) 330-342.

[7] A.K. Sengupta and B. Das, Maximum reach envelope for the seated and standing male and female for industrial workstation design. Ergonomics, 43 (2000) 1390-1404.

[8] M.B. Villanueva, H. Jonai and S. Saito, Ergonomic aspects of portable personal computers with flat panel displays (PCFPDs): evaluation of posture, muscle activities, discomfort and performance, Industrial Health, 36 (1998) 282-289. 